\title{
Article \\ Preliminary analysis of a Nano Relativistic Motor
}

\author{
Asher Yahalom ${ }^{1,2} \mathbb{1}$ *
}

1 Ariel University, Faulty of Engineering, Department of Electrical \& Electronic Engineering, Ariel 40700, Israel; asya@ariel.ac.il

2 Ariel University, Center for Astrophysics, Geophysics, and Space Sciences (AGASS), Ariel 40700, Israel;

* Correspondence: asya@ariel.ac.il; Tel. +972-54-7740294:

\begin{abstract}
Background; In a recent paper discussing Newton's third law in the framework of special relativity for charged bodies, it was suggested that one can construct a practical relativistic motor provided high enough charge and current densities are available. As on the macroscopic scale charge density is limited by the phenomena of dielectric breakdown, it was suggested to take advantage of the high charge densities which are available on the microscopic scale. (2) Methods; We use standard physical theories such as Maxwell electrodynamics and Quantum mechanics, supplemented by tools from vector analysis and numerics. (3) Results; We show that a hydrogen atom either in the ground state or excited state will not produce a relativistic engine effect, but by breaking the symmetry or putting the electron in a wave packet state may produce relativistic motor effect. (4) Conclusions; A highly localized wave packet will produce a strong relativistic motor effect. The preliminary analysis of the current paper suggests new promising directions of research both theoretical and experimental.
\end{abstract}

Keywords: Relativity; Nano Technology; Magnetic materials, Hydrogen, Symmetry Breaking, Relativistic Engine

\section{Introduction}

Relativity describes space-time structure. In Einstein's famous 1905 article: "On the Electrodynamics of Moving Bodies" [9] the theory was introduced for the first time. This result was a consequence of observations and electromagnetism laws, which were written in the middle of the nineteenth century by Maxwell in his famous differential equations [10-12] which owe their modern form to Oliver Heaviside [13]. Those equations imply that an electromagnetic wave travels at the speed of light $c$, which led the scientific community to believe that light is electromagnetic. Albert Einstein [9] used this to formulate his theory of relativity, which underlines that $c$ is the maximal velocity in nature. According to relativity, any object, message, signal (even if not electromagnetic), or field can not travel in as speed greater than the speed of light in vacuum. Thus retardation is established, if a phenomena occurs at a distance $R$ from an observer, it will not be noticed for at least a time of $\frac{R}{c}$. This means that action and reaction cannot be generated simultaneously because of propagation speed.

Newton's laws of motion, laid the foundation for modern science. These laws describe the relationship between the acting forces and motion in response to those forces. Three laws were first written by Isaac Newton in his Philosophiae Naturalis Principia Mathematica, first published in $1687[14,15]$. Here, we are concerned only with the third law, which states: When one body exerts a force on another body, the second body simultaneously exerts a force equal in magnitude and opposite in direction on the first body.

Due to Newton's third law, the sum of forces in a system which is not affected externally is zero. This law has a many experimental verifications and is a corner stone of physics. However, it is obvious that action and reaction cannot be generated at the same time because the speed of signal propagation is finite. Hence the third law cannot 
be correct, although it is valid for most practical applications due to high speed of signal propagation. Thus the total sum of forces cannot be zero. Still in the quasi-static approximation retardation may be neglected as was noted by D'Abramo [16] and also in section 3.1 of [20].

Current locomotive systems rely on interacting material parts; each gains momentum that is equal and opposite to the momentum obtained by its counterpart. A rocket ejects gas to propel itself forward. However, relativistic considerations suggest a new type of engine which is not derived from two material elements but of matter and field. It can be shown that the opposite amount of momentum to the one gained by the material body is gained by the field [4], thus the total momentum is indeed conserved. This follows from Noether's theorem which dictates that a system that possesses global translational symmetry will conserve linear momentum. A physical system composed of matter and field is invariant under translations, while every part (either matter or field) is not. Feynman [12] describes two orthogonally moving charges, apparently contradicting Newton's third law as forces that the charges induce do not cancel (last part of 26-2), this is solved in (27-6) in which it is noticed that the momentum gained by the two charge system is lost to the field.

A relativistic engine is defined as a system in which its material center of mass is in motion due to the interaction of its material components. Those parts may propagate with respect to each other or be fixed to a rigid frame. This is not relevant as we are interested only in the center of mass motion. We notice that a relativistic engine allows 3 -axis motion (vertical included), it does not contain moving parts, it does not consume fuel (and does not emit carbon) it only consumes electromagnetic energy which may be supplied by solar panels. The relativistic engine is a perfect for space travel in which much of the space vehicle volume is devoted to fuel storage. The energy can be put back into electromagnetic form once the vehicle reaches its destination indicating a very high potential efficiency.

Here we assume that the medium's magnetization and polarization are negligible and therefore we do not consider corrections to the Lorentz force suggested by [17]. Griffiths \& Heald [18] suggested that the laws of Coulomb's and Biot-Savart determine the electric and magnetic field configurations solely for static sources. Time-dependent generalizations of these laws described by Jefimenko [19] were used to study the applicability of Coulomb and Biot-Savart formulas outside the static domain. This was done also in the current paper and previous related papers.

In an earlier paper, we made use of Jefimenko's [11,19] equation to study the force developing between two current loops [1]. This was later expanded to include the forces between a current carrying loop and a permanent magnet [2,3]. Since the device is forced for a finite period, it will acquire momentum and energy in mechanical form. The question then arises if the law of momentum and energy conservation are violated. Linear momentum balance was discussed in [4]. The exchange of energy between the mechanical part of the relativistic engine and the electromagnetic field was studied in [5-8]. It was established that the electromagnetic energy expenditure is six times the kinetic energy gained by the relativistic motor. It was demonstrated that some energy might be radiated from the relativistic engine device if the coils are not configured correctly.

Previous analysis assumed bodies that were macroscopically natural. In a recent paper [20] we abandoned this assumption and analyzed charged bodies, thus revealing the consequences of charge on a possible electric relativistic engine. The charged relativistic engine allows to maintain a finite momentum even if the current is not continuously increasing which is not the case for the uncharged engine. However, due to the phenomena of dielectric breakdown which enforces charge density maximal values and the limitations on current density that can be transferred even through a superconducting wire it is concluded that for any reasonable geometrical size the momentum that can be gained in a relativistic charged engine is quite modest. 
It was thus suggested to take advantage of the high charge densities that are available in the microscopic realm, for example in ionic crystals. We will further pursue this idea in this paper in which we calculate the high charge densities and current densities in the atomic level. We will also deduce a preliminary form for the optimized wave function in terms of relativistic engine performance. We shall not derive the basic equations of the relativistic engine here the interested reader is referred to [20], only the principle results will be quoted.

\section{Main results regarding the charged relativistic motor}

Let us consider two charged sub systems having the charge densities $\rho_{1}, \rho_{2}$ and current densities $\vec{J}_{1}, \vec{J}_{2}$ then according to the analysis presented in [20] the following force is acting on the physical system composed of those two sub systems:

$$
\vec{F}_{T}^{[2]}=\frac{\mu_{0}}{4 \pi} \partial_{t} \iint d^{3} x_{1} d^{3} x_{2}\left[\frac{1}{2}\left(\rho_{2} \partial_{t} \rho_{1}-\rho_{1} \partial_{t} \rho_{2}\right) \hat{R}-\left(\rho_{1} \vec{J}_{2}+\rho_{2} \vec{J}_{1}\right) R^{-1}\right]
$$

in the above $\mu_{0}=4 \pi 10^{-7}$ is the vacuum permeability in MKS units, $\partial_{t}$ is a partial temporal derivative. $\int d^{3} x_{1}$ and $\int d^{3} x_{2}$ are integrals of the volume of the first and second subsystems respectively. We define $\vec{R}=\vec{x}_{1}-\vec{x}_{2}, R=|\vec{R}|, \hat{R}=\frac{\vec{R}}{R}$.

In the next subsections, we analyze examples of the implications of this formula. We remark that the it is based on a second order approximation, in some fast changing systems the second order correction will not be sufficient and higher order correction will be requited.

\subsection{Some basic preliminary observations}

According to Newton's second law, a system with a non zero total force in its center of mass, must have a change in its total linear momentum $\vec{P}(t)$ :

$$
\vec{F}_{T}^{[2]}=\frac{d \vec{P}}{d t}
$$

It can be shown that gravity alone has no "force" in free space and in case where gravity has a considerable effect the right hand side should be replaced by a geodesic equation of motion. However, in most cases electromagnetism is orders of magnitude stronger. Hence the right hand side should be considered a valid approximation to the geodesic form. Assuming that $\vec{P}(-\infty)=0$ and null current and charge densities at the same time, it follows that:

$$
\vec{P}(t)=\frac{\mu_{0}}{4 \pi} \iint d^{3} x_{1} d^{3} x_{2}\left[\frac{1}{2}\left(\rho_{2} \partial_{t} \rho_{1}-\rho_{1} \partial_{t} \rho_{2}\right) \hat{R}-\left(\rho_{1} \vec{J}_{2}+\rho_{2} \vec{J}_{1}\right) R^{-1}\right]
$$

Thus we obtain finite linear momentum for stationary charge and current densities:

$$
\vec{P}(t)=-\frac{\mu_{0}}{4 \pi} \iint d^{3} x_{1} d^{3} x_{2}\left(\rho_{1} \vec{J}_{2}+\rho_{2} \vec{J}_{1}\right) R^{-1}
$$

it follows that the charged relativistic engine can produce forward linear momentum without interacting with any external system except it own electromagnetic field. The above expression can be simplified using the instantaneous (non retarded) potentials:

$$
\Phi_{1}\left(\vec{x}_{2}\right)=\frac{1}{4 \pi \epsilon_{0}} \int d^{3} x_{1} \frac{\rho_{1}\left(\vec{x}_{1}, t\right)}{R}, \quad \Phi_{2}\left(\vec{x}_{1}\right)=\frac{1}{4 \pi \epsilon_{0}} \int d^{3} x_{2} \frac{\rho_{2}\left(\vec{x}_{2}, t\right)}{R}
$$

$\epsilon_{0}=8.8510^{-12}$ is the vacuum permittivity in MKS units. Using equation (5) we may write:

$$
\vec{P}(t)=-\frac{1}{c^{2}}\left[\int d^{3} x_{2} \Phi_{1} \vec{J}_{2}+\int d^{3} x_{1} \Phi_{2} \vec{J}_{1}\right]
$$


in which we are reminded that $\mu_{0} \epsilon_{0}=\frac{1}{c^{2}}$. Another important result is that in a charged relativistic motor we do not need both subsystems to be charged, that is we can take $\rho_{2}=0$ :

$$
\vec{P}(t)=-\frac{\mu_{0}}{4 \pi} \iint d^{3} x_{1} d^{3} x_{2} \rho_{1} \vec{J}_{2} R^{-1}=-\frac{1}{c^{2}} \int d^{3} x_{2} \Phi_{1} \vec{J}_{2}
$$

provided that the system has a non vanishing current density $\vec{J}_{2}$. The same result will be obtained if sub systems 1 and 2 are charged but system 1 lacks current density: $\vec{J}_{1}=0$.

In [4] we showed that the forward linear momentum gained by the mechanical system will be balanced by a backward linear momentum gained by the electromagnetic system. We now study the challenges involved in constructing a powerful charged relativistic engine.

\subsection{Macroscopic charge density limitations}

The charge that can be put in a volume or two dimensional surface is restricted due to the phenomena of electrical breakdown. In this case the surrounding medium is separated into electron and ions and becomes a conducting plasma. Thus a discharge results and the charge density is reduced and even nullified. The typical dielectric strength $E_{\text {max }}$ of air is $3 \mathrm{MV} / \mathrm{m}$ [21], for high vacuum one can achieve 20-40 MV/m [22] and for a diamond $2000 \mathrm{MV} / \mathrm{m}$ [23]. For an infinite surface the surface density $\sigma$ is:

$$
\sigma=2 \epsilon E<\sigma_{\max }=2 \epsilon E_{\max }, \quad \epsilon=\epsilon_{r} \epsilon_{0}
$$

in which $\epsilon_{r}$ is the relative susceptibility. For air $\sigma_{\max } \simeq 53 \mu \mathrm{C} / \mathrm{m}^{2}$. To calculate the charge $Q$ which one can maintain in a given volume we notice that for a spherical symmetric charge ball we obtain at a distance $r$ the radial field $E$ :

$$
E=\frac{k Q}{r^{2}}, \quad k=\frac{1}{4 \pi \epsilon_{0}}
$$

the stronger field is on the ball itself, that is at $r=r_{s}$ we must have:

$$
\frac{k Q}{r_{s}^{2}}<E_{m} \Rightarrow Q<Q_{m}=\frac{1}{k} r_{s}^{2} E_{m}
$$

For a size of $1 \mathrm{~m}$, the maximal charge is $3.310^{-4} \mathrm{C}$. Hence, regardless if we have surface charge or a volume charge the maximal charge scales as the square of the dimension of the system, that is as $h^{2}$. A possible approach to increase the available charge density is to use electret. Fluorinated parylene (Parylene HT, SCS) offers excellent surface charge density of $3.7 \mathrm{mC} / \mathrm{m}^{2}$ found for a $7.3 \mu \mathrm{m}$ film by Hsi-wen and Yu-Chong [24], this material has a dielectric strength of $204.58 \mathrm{MV} / \mathrm{m}$. However, as the thickness of the material grows the charge density is less.

\subsection{Macroscopic current density limitations}

The current a device can generate depends on its voltage difference and internal resistance. Provided that the external impedance is insignificant, the resulting currents bcoeme short currents and may be of the order of a few thousand amperes for a standard domestic electrical installation and as high as hundreds of thousands of amperes in industrial power installations. Current flowing through a metal conductor will generate heat due to finite resistivity. Large currents require a thick conductor to avoid dangerous heating. This shortcoming can be circumvented using a superconductor, however, this will require cooling to low temperatures, making the system cumbersome. But, even a superconductor has a critical current density and superconduction properties losses above that. Jung, S. G. et al. [25] have reported critical current densities as high as $5 \mathrm{kA} / \mathrm{cm}^{2}$. Coil windings will enable to reuse the current, and thus the winding number in a given area is critical for performance. Proximity of current and charge will affect the 
amount of generated linear momenta according to equation (7). However, installing a conductor close to the charge may result in discharge, hence a balance is required.

\subsection{Scalability}

It is obvious from equation (7) that a larger relativistic motor is more powerful. However, since the charge surface density is limited the linear momentum gained will scale as the second power of the dimension of the charged subsystem and as the third power of the dimension of the current carrying system.

\subsection{Energy}

An engine of mass $M$ will acquire a kinetic energy of:

$$
E_{k}=\frac{\vec{P}^{2}}{2 M}
$$

$\vec{P}$ is calculated according to equation (3). The energy needed for the engine may be bigger or equal to the kinetic energy of the engine even in ideal cases were losses due to drag, friction and ohmic resistance are neglected. For a non charged relativistic motor the energy removed from the total electromagnetic energy is equal to $E=6 E_{k}$ [8], thus the energy consumed by a charged relativistic motor should be of the order of magnitude of the kinetic energy given in equation (11) (but probably larger). The power needed is $\frac{d E}{d t}$, thus according to equation (3) it will depend on the rise time of the current. Finally we notice that in an ideal situation in which drag, viscosity losses (space travel) and ohmic losses (superconducting wires)are neglected the efficiency of the relativistic engine is infinite, and the entire mechanical energy can be converted back to electromagnetic energy as the device comes to full stop. This idea is partially implemented in today hybrid cars, in which pressing the brakes converts the kinetic energy of the car to electromagnetic energy stored in a battery.

Notice also that if not configured correctly a relativistic motor may radiate. This was shown in previous works for an uncharged relativistic motor $[7,8]$. The total force generation given by equation equation (1) includes also radiating field configurations, hence in this respect it will not affect the reported results.

\section{The nano relativistic engine}

In a previous paper [20] we have shown that intrinsic parameter limitations, especially dielectric breakdown lead to somewhat modest amount of momenta that can be gained by relativistic motor. However, it seams that in the microscopic domain this limitation is rather relaxed. For example if consider ionic crystals such as the prevalent table salt: $\mathrm{Na}^{+} \mathrm{Cl}^{-}$. This salt solidifies to a face centered cubic lattice in which the lattice constant is $l=564.02 \mathrm{pm}$. Taking for example the 100 plane of this lattice (see Figure 1) we can see that the charge density is periodical in which in each half unit cell we have a surface charge density of \pm 2 Coulomb $/ \mathrm{m}^{2}$. This is of course thousand time larger than available macroscopic charge densities (see section 2.2), however, on the macroscopic average this leads to a null charge density. We also notice that a smaller lattice constant will cause a still higher charge density, but it seems that the lattice constant cannot be smaller than the relevant atom hence it is bounded from below. 


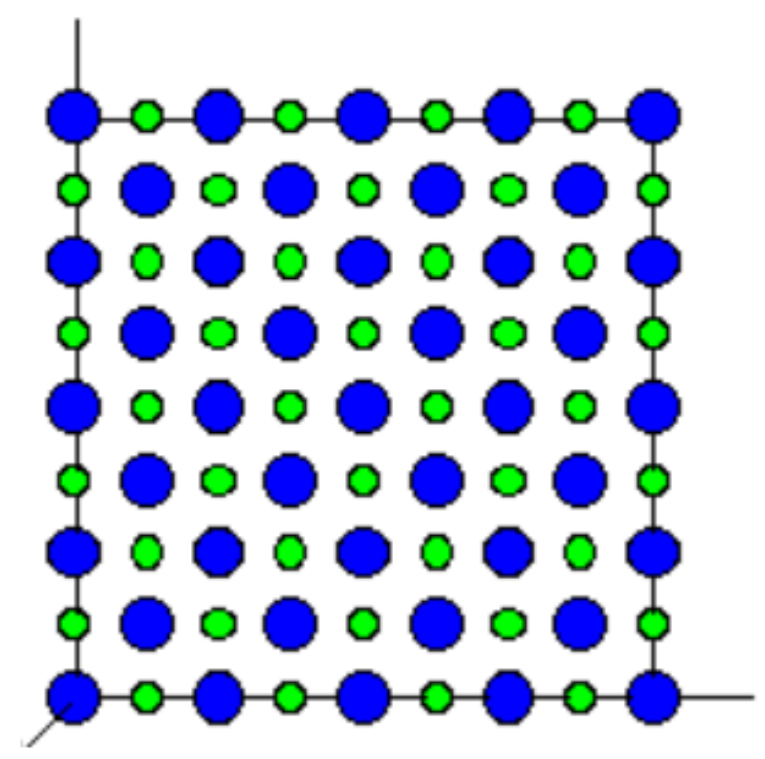

Figure 1. The 100 plane of a table salt $\mathrm{Na}^{+} \mathrm{Cl}^{-}$lattice, blue circles stand for Sodium positive ions and yellow circles stand for Chlorine negative ions.

To see how we can circumvent this unfortunate reality we will investigate equation ( 7), calculating the spatial Fourier transform of the scalar potential and the current density such that:

$$
\begin{aligned}
\Phi_{1}(\vec{k}) & =\int_{-\infty}^{+\infty} \int_{-\infty}^{+\infty} \int_{-\infty}^{+\infty} \Phi_{1}(\vec{x}) e^{-i \vec{k} \cdot \vec{x}} d^{3} x \\
\vec{J}_{2}(\vec{k}) & =\int_{-\infty}^{+\infty} \int_{-\infty}^{+\infty} \int_{-\infty}^{+\infty} \vec{J}_{2}(\vec{x}) e^{-i \vec{k} \cdot \vec{x}} d^{3} x
\end{aligned}
$$

Using the theorem of Parseval [26], we may now write equation (7) in the form:

$$
\vec{P}(t)=-\frac{1}{c^{2}(2 \pi)^{3}} \int_{-\infty}^{+\infty} \int_{-\infty}^{+\infty} \int_{-\infty}^{+\infty} d^{3} k \Phi_{1}^{*}(\vec{k}) \vec{J}_{2}(\vec{k})
$$

In this form it is obvious, that a microscopic distribution of periodic charge density will be beneficial if it is a accompanied by a current density distribution of the same period. How can we obtain such a microscopic charge density distribution? To this end we remind the reader that microscopic currents are associated with the electronic motion and electronic spin. The magnetization $\vec{M}$ is related to the magnetization current $\vec{J}_{M}$ by the formulae [2,3]:

$$
\vec{J}_{M} \equiv \vec{\nabla} \times \vec{M}
$$

We may replace in equation (6), equation (7) and equation (13) $\vec{J}$ by $\vec{J}_{M}$ to obtain a similar effect. Furthermore, the magnetization $\vec{M}$ is related to a the microscopic dipole moments $\vec{m}_{i}$ through:

$$
\vec{M} \equiv \frac{1}{V} \sum_{i} \vec{m}_{i}
$$

in which we sum over all dipoles and divide by the sample volume $V$. In known magnetic materials for example iron, magnetic dipole moments are a consequence of the atom spin configuration. For example, in the case of $\alpha$-iron the spins of two unpaired electrons in each atom generally align with the spins of its closest neighbors. The reason for this is that the orbitals of those two electrons $\left(d_{z^{2}}\right.$ and $\left.d_{x^{2}-y^{2}}\right)$ do not point in the direction of neighboring atoms in the lattice, and since this is the case are not involved in 
metallic bonding. We thus hypothesize that the ideal structure of a relativistic motor will involve an ionic lattice in which one species of atom (say the positively charged) will involve free spins that can be manipulated by an external magnetic field thus creating a relativistic engine effect which can be easily manipulated in three axis. In the next section we discuss the properties of a relativistic engine in the microscopic scale.

\section{Relativistic engine in the microscopic scale}

In the microscopic scale relevant to the relativistic engine to be discussed here matter is composed of electron and nuclei which are put together to become natural atoms, ions, molecules and lattice structures. There are of course smaller scales which one can discuss which are relevant to nuclear physics, elementary particles etc. but this will not concern us here. Matter at the microscopic scale is described by quantum mechanics. In this preliminary work it will suffice to discuss a one electron system in which the nuclei effecting the motion of the electron are modeled as point charges.

\subsection{Schrödinger's electron}

Schrödinger's electron is an electron in which the spin property is ignored. The earliest appearance of the non-relativistic probability density and current density satisfying a continuity equation is due to Schrödinger himself [27], obtained from his time-dependent wave-equation:

$$
i \hbar \dot{\psi}=\hat{H} \psi, \quad \hat{H}=-\frac{\hbar^{2}}{2 m} \vec{\nabla}^{2}+V
$$

in the above $i=\sqrt{-1}$ and $\psi$ is the complex wave function. $\dot{\psi}=\frac{\partial \psi}{\partial t}$ is the partial time derivative of the wave function. $\hbar=\frac{h}{2 \pi}$ is Planck's constant divided by $2 \pi$ and $m$ is the particles mass, $V$ is the potential of a force acting on the particle. If now the modulus $a$ and phase $\phi$ are introduced through:

$$
\psi=a e^{i \phi}
$$

The following continuity equation is satisfied provided that $\psi$ satisfies equation (16):

$$
\frac{\partial \tilde{\rho}}{\partial t}+\vec{\nabla} \cdot \vec{J}_{p}=0
$$

in which the probability density is defined as:

$$
\tilde{\rho}=a^{2}
$$

and is of course normalized, that is:

$$
\int \tilde{\rho} d^{3} x=1
$$

The probability current density is:

$$
\vec{J}_{p}=\frac{\hbar}{m} \tilde{\rho} \vec{\nabla} \phi
$$

As the charge density of an electron with charge $-e$ is:

$$
\rho=-e \tilde{\rho}
$$

it follows that the current density is:

$$
\vec{J}=-e \vec{J}_{p}=-\frac{\hbar e}{m} \tilde{\rho} \vec{\nabla} \phi=\frac{\hbar}{m} \rho \vec{\nabla} \phi,
$$


Satisfying the charge density continuity equation:

$$
\frac{\partial \rho}{\partial t}+\vec{\nabla} \cdot \vec{J}=0
$$

\subsection{Pauli's electron}

Schrödinger's quantum mechanics is limited to the description of spin less particles. If spin is to be taken into account we must use the Pauli equation (for a non-relativistic particle) :

$$
i \hbar \dot{\psi}=\hat{H} \psi, \quad \hat{H}=-\frac{\hbar^{2}}{2 m}\left[\vec{\nabla}-\frac{i e}{\hbar c} \vec{A}\right]^{2}+\mu \vec{B} \cdot \vec{\sigma}+V
$$

$\psi$ here is a two dimensional complex column vector (also denoted as spinor), $\hat{H}$ is a two dimensional hermitian operator matrix, $\mu$ is the magnetic moment of the particle, $c$ is the velocity of light in vacuum. The electromagnetic interaction is described by the vector $\vec{A}$ and scalar $V$ potentials and the magnetic field $\vec{B}=\vec{\nabla} \times \vec{A} . \vec{\sigma}$ is a vector of two dimensional Pauli matrices which can be represented as follows:

$$
\sigma_{1}=\left(\begin{array}{cc}
0 & 1 \\
1 & 0
\end{array}\right), \quad \sigma_{2}=\left(\begin{array}{cc}
0 & -i \\
i & 0
\end{array}\right), \quad \sigma_{3}=\left(\begin{array}{cc}
1 & 0 \\
0 & -1
\end{array}\right)
$$

A spinor $\psi$ satisfying equation (25) must also satisfy a continuity equation of the form:

$$
\frac{\partial \tilde{\rho}}{\partial t}+\vec{\nabla} \cdot \vec{J}_{p}=0
$$

In the above:

$$
\tilde{\rho}=\psi^{\dagger} \psi, \quad \vec{J}_{p}=\frac{\hbar}{2 m i}\left[\psi^{\dagger} \vec{\nabla} \psi-\left(\vec{\nabla} \psi^{\dagger}\right) \psi\right]-\frac{e}{m c} \vec{A} \tilde{\rho} .
$$

The symbol $\psi^{\dagger}$ represents a row spinor (the transpose) whose components are equal to the complex conjugate of the column spinor $\psi$. Holland [28] has suggested the following representation of the spinor:

$$
\psi=R e^{i \frac{\chi}{2}}\left(\begin{array}{c}
\cos \left(\frac{\theta^{\prime}}{2}\right) e^{i \frac{\phi^{\prime}}{2}} \\
i \sin \left(\frac{\theta^{\prime}}{2}\right) e^{-i \frac{\phi^{\prime}}{2}}
\end{array}\right) \equiv\left(\begin{array}{c}
\psi_{\uparrow} \\
\psi_{\downarrow}
\end{array}\right) .
$$

In terms of this representation the probability density is given as:

$$
\tilde{\rho}=\psi^{\dagger} \psi=R^{2} \Rightarrow R=\sqrt{\tilde{\rho}} .
$$

The charge density is given as:

$$
\rho=-e \tilde{\rho}=-e \psi^{\dagger} \psi=-e R^{2} .
$$

The spin density can be calculated using the representation given in equation (29) as:

$$
\hat{s} \equiv \frac{\psi^{+} \vec{\sigma} \psi}{\rho}=\left(\sin \theta^{\prime} \sin \phi^{\prime}, \sin \theta^{\prime} \cos \phi^{\prime}, \cos \theta^{\prime}\right)
$$

This gives an easy physical interpretation to the variables $\theta^{\prime}, \phi^{\prime}$ as angles which describe the projection of the spin density on the axes. $\theta^{\prime}$ is the elevation angle of the spin density vector and $\phi^{\prime}$ is the azimuthal angle of the same. The probability current density can now be calculated by inserting $\psi$ given in equation (29) into equation (28):

$$
\vec{J}_{p}=\frac{\hbar}{2 m} \tilde{\rho}\left(\vec{\nabla} \chi+\cos \theta^{\prime} \vec{\nabla} \phi^{\prime}\right)-\frac{e}{m c} \tilde{\rho} \vec{A} .
$$


if the vector potential contribution is not significant, we may write:

$$
\vec{J}_{p}=\frac{\hbar}{2 m} \tilde{\rho}\left(\vec{\nabla} \chi+\cos \theta^{\prime} \vec{\nabla} \phi^{\prime}\right)
$$

Hence, the electric current density is:

$$
\vec{J}=-e \vec{J}_{p}=-\frac{e \hbar}{2 m} \tilde{\rho}\left(\vec{\nabla} \chi+\cos \theta^{\prime} \vec{\nabla} \phi^{\prime}\right)=\frac{\hbar}{2 m} \rho\left(\vec{\nabla} \chi+\cos \theta^{\prime} \vec{\nabla} \phi^{\prime}\right) .
$$

If the electron is in a definitive spin state (say spin up) and the vector potential is null, equation (25) is the same as equation (16). Thus for preliminary order of magnitude estimations Schrödinger equation solutions will suffice.

\subsection{The Hydrogen atom}

The Hydrogen atom is one of the simplest quantum mechanical systems, and one of the few quantum mechanical systems that can be solved exactly. The Hydrogen atom is composed of a proton and an electron, the proton is modeled as a positive point charge of charge $+e$ located conveniently at the origin of the axis. $V$ in equation (16) is thus equal to $-e \Phi$ which is felt by an electron of charge $-e$, in which $\Phi$ is given in equation ( 5 ) for a positive point charge $+e$ located at the origin of axis, that is:

$$
V=-e \Phi=-\frac{k e^{2}}{r}
$$

The electron wave function can be of the form:

$$
\psi=e^{-\frac{i E_{n} t}{\hbar}} \psi_{n}, \quad \hat{H} \psi_{n}=E_{n} \psi_{n}
$$

In which $\psi_{n}$ is a spatial eigenfunction of the Hamiltonian $\hat{H}$ with eigenenergy $E_{n}$. The electron can be in a definite energy state or in a superposition of states. The functional form of $\psi_{n}$ is well known and is given in terms of the spherical coordinates $r, \theta, \varphi$ as follows:

$$
\psi_{n l m}=\sqrt{\left(\frac{2}{n a_{0}}\right)^{3} \frac{(n-l-1) !}{2 n(n+l) !}} e^{-\frac{r^{\prime}}{2}} r^{\prime l} L_{n-l-1}^{2 l+1}\left(r^{\prime}\right) Y_{l}^{m}(\theta, \varphi) .
$$

In the above $L_{n-l-1}^{2 l+1}$ is the generalized Laguerre polynomial of degree $n-l-1$, and $Y_{l}^{m}(\theta, \varphi)$ is a spherical harmonic function of degree $l$ and order $m$ defined as:

$$
Y_{l}^{m}(\theta, \varphi)=(-1)^{m} \sqrt{\frac{2 l+1}{4 \pi} \frac{(l-m) !}{(l+m) !}} P_{l}^{m}(\cos \theta) e^{i m \varphi} .
$$

In which $P_{l}^{m}$ are associated Legendre polynomials. The definition also contains the reduced Bohr radius:

$$
a_{0} \equiv \frac{4 \pi \epsilon_{0} \hbar^{2}}{m_{e}^{\prime} e^{2}} \simeq \frac{\hbar^{2}}{k m_{e} e^{2}} \simeq 0.5310^{-10} \mathrm{~m}, \quad m_{e}^{\prime} \equiv \frac{m_{e} m_{p}}{m_{e}+m_{p}} \simeq m_{e}
$$

in which $m_{e}$ and $m_{p}$ are the masses of the electron and proton respectively. Finally we use a normalized radial coordinate $r^{\prime}$ defined as:

$$
r^{\prime} \equiv \frac{2 r}{n a_{0}}
$$

The eigen energies defined in equation (37) are functions of the principal quantum number $n=1,2,3 \ldots$ :

$$
E_{n}=-\frac{m_{e}^{\prime} e^{4}}{32 \pi^{2} \epsilon_{0}^{2} \hbar^{2}} \frac{1}{n^{2}}
$$


Hence the function $\psi_{n l m}$ are degenerate in the sense that different functions have the same energy, the degeneracy can be lifted if there is a perturbation that changes the potential $V$ to a form that is not spherically symmetric. For a given energy the different eigen functions are listed by their azimuthal quantum number $l=0,1,2, \ldots, n-1$ and their magnetic quantum number $m=-l, \ldots, l$. Notice that the amplitude of the wave function given in equation (37) is a function of $r$ and $\theta$ only, but not of $\varphi$ and the time $t$.

$$
a(r, \theta)=|\psi|=\left|\psi_{n l m}\right| \Rightarrow \rho=-e\left|\psi_{n l m}\right|^{2} .
$$

Notice, also that the phase is a function of time and $\varphi$ but not of $r$ and $\theta$ :

$$
\phi(\varphi, t)=m(\varphi+\pi)-\frac{E_{n} t}{\hbar} .
$$

We are now in a position to calculate the current density given in equation (23)

$$
\vec{J}=\frac{\hbar}{m_{e}} \rho \vec{\nabla} \phi=-m \frac{e \hbar}{m_{e}}\left|\psi_{n l m}\right|^{2} \frac{\hat{\varphi}}{r \sin \theta}
$$

(the reader should not confuse the magnetic number $m$ with the mass $m$ used in previous sections). In the above we have used the spherical representation of the nabla operator which is given in terms of the unit vectors $\hat{r}, \hat{\theta}, \hat{\varphi}$ as:

$$
\vec{\nabla}=\hat{r} \frac{\partial}{\partial r}+\frac{\hat{\theta}}{r} \frac{\partial}{\partial \theta}+\frac{\hat{\varphi}}{r \sin \theta} \frac{\partial}{\partial \varphi} .
$$

We notice that the current density is linear in the magnetic number $m$, in particular if $m=0$ there is no current density and thus no relativistic motor effect. We conclude that for an isolated hydrogen in the ground state $n=1, l=0, m=0$ there is no relativistic motor effect. But also in excited states in which the current density does not necessarily vanish there will be no relativistic motor effect if the potential acting on the electron is spherically or cylindrically symmetric as is evident from equation (7). To see this notice that:

$$
\hat{\varphi}=-\sin \varphi \hat{x}+\cos \varphi \hat{y}
$$

$\hat{x}$ and $\hat{y}$ are constant unit vectors in the $x$ and $y$ directions respectively. In spherical coordinates the volume element is:

$$
d^{3} x=r^{2} \sin \theta d r d \theta d \varphi
$$

since $\Phi\left|\psi_{n l m}\right|^{2}$ is cylindrically symmetric it does depend on $\varphi$ and the result follows immediately. We also notice that apparent singular terms in the current density of the form $\frac{1}{r \sin \theta}$ (see equation (45)) are cancelled out by identical terms in the volume element.

How can we use an hydrogen atom as a component in a relativistic motor despite the fact that it is useless either in the ground state or in an excited state? In the following section we will suggest two approaches, in one the electron is not in an energy eigen state but in a superposition of states and in the other the potential is not cylindrically symmetric. Both approaches will yield a finite relativistic engine effect.

But before we proceed we make some orders of magnitude estimates to justify our endeavour. The volume charge density of an electron in a hydrogen atom has an order of magnitude:

$$
\bar{\rho}=\frac{e}{\frac{4}{3} \pi a_{0}^{3}} \simeq 2.610^{11} \text { Coloumb } / \mathrm{m}^{3} .
$$

The surface charge density of an electron in a hydrogen atom has an order of magnitude:

$$
\bar{\sigma}=\frac{e}{4 \pi a_{0}^{2}} \simeq 4.5 \text { Coloumb } / \mathrm{m}^{2} .
$$


this is of the same order of magnitude as we obtained for the salt crystal in section 3, and a thousand times larger than what is available for macroscopic charge densities (see section 2.2). The order of magnitude of the current density is:

$$
\bar{J}=\frac{e \hbar}{m_{e} a_{0}^{4}} \simeq 2.310^{18} \text { Ampere } / \mathrm{m}^{2}=2.310^{14} \text { Ampere } / \mathrm{cm}^{2}
$$

This current density is ten orders of magnitude larger than what can be achieved in a macroscopic scale (see section 2.3). Although in the Copenhagen interpretation of quantum mechanics an electron does not have a trajectory and therefore does not have a velocity we can still associate with the current density a typical velocity:

$$
\bar{v}=\frac{\bar{J}}{\bar{\rho}} \sim 9.110^{6} \mathrm{~m} / \mathrm{s}=0.03 \mathrm{c}
$$

Hence the associated velocity is rather large although still significantly smaller than the speed of light.

\subsection{A wave packet of Hydrogen atom eigenstates}

Let us assume an idealized wave packet of the form:

$$
\psi=A e^{i k^{\prime} x}, \quad A=\left\{\begin{array}{cc}
\sqrt{\tilde{\rho}_{c}} & r<R_{\max } \\
0 & r \geq R_{\max }
\end{array}\right.
$$

in the above $k^{\prime}$ and $\tilde{\rho}_{c}$ are constants. As the wave function must be normalized it follows from equation (20) that $\tilde{\rho}_{c}$ must take the following value:

$$
\tilde{\rho}_{c}=\frac{3}{4 \pi} R_{\max }^{-3}
$$

hence this wave function has a linear phase and a uniform amplitude which is confined inside a sphere of radius $R_{\max }$. It is certainly not an eigen state of the Hydrogen atom Hamiltonian but may be approximated by a superposition of the eigenstates:

$$
\psi \simeq \sum_{n l m} a_{n l m}(t) \psi_{n l m}
$$

The desired functions $a_{n l m}(t)$ can be calculated using the orthonormality properties of the eigen functions:

$$
a_{n l m}(t)=<\psi_{n l m} \mid \psi>=\int d^{3} x \psi_{n l m}^{*} \psi
$$

the preparation of such a state will require a suitable electromagnetic pulse in accordance with equation (25), the shape of the pulse for creating and maintaining the wave packet is beyond the scope of the current paper.

We can now calculate the current density using equation (23) as follows:

$$
\vec{J}=-\frac{\hbar e}{m_{e}} \tilde{\rho} \vec{\nabla} \phi=-\frac{\hbar k e}{m_{e}} \tilde{\rho}_{c} \hat{x}=-e v \tilde{\rho}_{c} \hat{x} \quad \text { for } \quad r<R_{\max }, \quad v \equiv \frac{\hbar k^{\prime}}{m_{e}},
$$

$v$ has the units of velocity. Let us now take system 1 in equation (7) to be the proton and system 2 to be electron. The proton is conveniently modelled as a point charge located at the origin of axis. That is:

$$
\rho_{1}=e \delta^{(3)}\left(\vec{x}_{1}\right)
$$

It thus follows:

$$
\vec{P}(t)=-\frac{\mu_{0}}{4 \pi} \iint d^{3} x_{1} d^{3} x_{2} \rho_{1} \vec{J}_{2} R^{-1}==-\frac{\mu_{0} e}{4 \pi} \int d^{3} x_{2} \vec{J}_{2} r_{2}^{-1} .
$$


Plugging equation (48) and equation (57) into the above expression and integrating will result in:

$$
\vec{P}(t)=\frac{1}{2} \mu_{0} e^{2} v \tilde{\rho}_{c} R_{m a x}^{2} \hat{x} .
$$

Taking into account normalization according to equation (54) we obtain the following expression for $\vec{P}(t)$ :

$$
\vec{P}(t)=\frac{3}{8 \pi} \mu_{0} e^{2} v R_{\text {max }}^{-1} \hat{x} .
$$

It follows that the momentum gained by a relativistic motor is linearly proportional to the electron's "velocity" $v$ and inversely proportional to the wave packet spatial extension $R_{\text {max }}$. As the maximal speed generated by the hydrogen relativistic motor is obtained when the engine is not loaded and need to carry only its own mass it follows that:

$$
v_{\max }=\frac{P}{m_{p}}=\frac{3}{8 \pi m_{p}} \mu_{0} e^{2} v R_{\max }^{-1} .
$$

the electron mass $m_{e}$ is ignored as it is much smaller than the proton mass $m_{p}$. Thus to obtain a predefined velocity $v_{\max }$ we need a wave packer of the radius:

$$
R_{\max }=\frac{3 \mu_{0} e^{2} v}{8 \pi m_{p} v_{\max }}
$$

According to equation (52) the typical "velocity" of the electron is $0.03 c$ in the hydrogen atom. Here we will assume that $v \simeq c$ for the purpose of obtaining the maximal $R_{\max }$ required, taking into account that in reality we will need a smaller $R_{\max }$ to achieve the desired velocity. We also remark that for a relativistic electron the Schrödinger formalism is not adequate and one should use a Dirac equation instead. Moreover, the relativistic engine considered so far assumes slow moving components where the relativistic effect is due to the retardation of the electromagnetic signal, a different mathematical treatment is needed if the components of the engine move with relativistic speeds. Taking this into account we make the following preliminary observations: for a typical car a velocity of $v_{\max }=50 \mathrm{~m} / \mathrm{s}=180 \mathrm{~km} / \mathrm{h}$ we obtain:

$$
R_{50} \simeq 1.410^{-11} \mathrm{~m}=0.26 a_{0}
$$

hence the wave packet is about quarter of the atomic size. If we need the hydrogen relativistic engine to reach the earth's escape velocity of $v_{\max }=11.2 \mathrm{~km} / \mathrm{s}$ it follows that:

$$
R_{\text {escape velocity }} \simeq 6.110^{-14} \mathrm{~m} \simeq 10^{-3} a_{0} \simeq 73 r_{p}
$$

in which $r_{p}=8.410^{-16} \mathrm{~m}$ is the proton charge radius. Thus for such velocities the wave packet is of a typical nuclear size rather than an atomic size. Finally if we imagine that the relativistic engine will reach the maximal speed available in a Lorentzian space-time for a particle which is initially subluminal that is $v_{\max } \simeq c$ it follows that:

$$
R_{\text {light speed }} \simeq 2.310^{-18} \mathrm{~m} \simeq 310^{-3} r_{p}
$$

that is the wave packet must be of sub nuclear dimensions.

We conclude this section by calculating the standard deviation of the wave packet defined in equation (53).

$$
\sigma_{x}^{2}=\int d^{3} x(x-E[x])^{2}|\psi|^{2}=\int d^{3} x(x-\bar{x})^{2} A^{2}
$$

In which the expectation value of position is:

$$
E[x]=\int d^{3} x x|\psi|^{2}=\int d^{3} x x A^{2}=0
$$


It follows thus that:

$$
\sigma_{x}^{2}=\int d^{3} x x^{2} A^{2}=\frac{1}{5} R_{\max }^{2} \quad \Rightarrow \quad \sigma_{x}=\frac{1}{\sqrt{5}} R_{\max }
$$

Similarly:

$$
E[y]=E[z]=0, \quad \sigma_{y}=\sigma_{z}=\frac{1}{\sqrt{5}} R_{\max }
$$

Thus the electron is expected to be found in coincidence with the proton with a standard deviation which is about half the size of the wave packet. Calculating the expectation value for the momentum operators $\hat{p}_{x}=-i \hbar \frac{\partial}{\partial x}$ we find that:

$$
E\left[p_{x}\right]=\int d^{3} x \psi^{*} \hat{p}_{x} \psi=-i \hbar \int d^{3} x \psi^{*} \frac{\partial \psi}{\partial x}=\hbar k^{\prime}
$$

similarly:

$$
\begin{aligned}
& E\left[p_{y}\right]=\int d^{3} x \psi^{*} \hat{p}_{y} \psi=-i \hbar \int d^{3} x \psi^{*} \frac{\partial \psi}{\partial y}=0, \\
& E\left[p_{y}\right]=\int d^{3} x \psi^{*} \hat{p}_{y} \psi=-i \hbar \int d^{3} x \psi^{*} \frac{\partial \psi}{\partial y}=0
\end{aligned}
$$

hence the expected velocity of the electron is equal to the current velocity defined in equation (57) and in the same direction:

$$
v=\frac{E\left[p_{x}\right]}{m_{e}}=\frac{\hbar k^{\prime}}{m_{e}}
$$

The standard deviation of the momentum operator in any direction is infinite due to the ideal discontinues form of the wave function, meaning that when measuring the electron velocity any value can be obtained with non vanishing probability. We notice, however, that even for a more realistic and smooth wave packet we expect a non vanishing standard deviation of the electron momenta due to the Heisenberg uncertainty relation:

$$
\sigma_{p_{x}} \geq \frac{\hbar}{2 \sigma_{x}} \simeq \frac{\hbar}{R_{\max }}, \quad \sigma_{p_{y}} \geq \frac{\hbar}{2 \sigma_{y}} \simeq \frac{\hbar}{R_{\max }}, \quad \sigma_{p_{z}} \geq \frac{\hbar}{2 \sigma_{z}} \simeq \frac{\hbar}{R_{\max }}
$$

hence the measured velocity of the electron may differ significantly from the expected current velocity.

\subsection{Symmetry breaking}

As pointed out earlier the relativistic engine effect will be null for an hydrogen atom in either the ground or excited state due to the symmetry of the proton potential. However, if the symmetry is broken the relativistic engine effect is recovered. We thus assume an additional proton located at a distance $d$ from the hydrogen atom. This proton will break the cylindrical symmetry. In what follows we will consider system 1 as the additional proton and system 2 will be the electron. We will assume that the proton is at a distance $d$ from the axis origin in the negative $x$ direction. The potential generated by this additional proton locate at $\vec{x}_{1}=-d \hat{x}$ is according to equation (5):

$$
\Phi_{1}\left(\vec{x}_{2}\right)=\frac{k e}{\left|\vec{x}_{1}-\vec{x}_{2}\right|}=\frac{k e}{\left|-d \hat{x}-\vec{x}_{2}\right|}=\frac{k e}{\sqrt{d^{2}+\left|\vec{x}_{2}\right|^{2}+2 d x_{2}}} .
$$


From now on we drop the index 2 and use standard spherical coordinates. Hence $r=\left|\vec{x}_{2}\right|$ and $x_{2}=r \sin \theta \cos \varphi$, thus:

$$
\Phi_{1}(\vec{x})=\frac{k e}{\sqrt{d^{2}+r^{2}+2 d r \sin \theta \cos \varphi}} .
$$

the potential $\varphi$ dependence clearly demonstrates the symmetry breaking of the above potential. We can now calculate the relativistic motor momentum defined in equation (7) by inserting the potential of equation (76) and the current obtained in equation (45) for an electron in an eigen state of the hydrogen atom:

$$
\vec{P}=\frac{\mu_{0}}{4 \pi} e \bar{J} m a_{0} \int_{0}^{\infty} r d r \int_{0}^{\pi} d \theta \int_{0}^{2 \pi} d \varphi \frac{A^{\prime 2}}{\sqrt{d^{2}+r^{2}+2 d r \sin \theta \cos \varphi}}(-\sin \varphi \hat{x}+\cos \varphi \hat{y}) .
$$

in which $\bar{J}$ is given in equation (51), $m$ is the magnetic quantum number (not the mass), and $\hat{\varphi}$ is given through equation (47). Finally:

$$
A^{\prime}(r, \theta)=\left|\psi_{n l m}\right| a_{0}^{\frac{3}{2}}
$$

is the dimensionless state amplitude in which we suppress the quantum indices $\mathrm{nlm}$. The above expression can be somewhat simplified as follows:

$$
\vec{P}=\frac{\mu_{0}}{4 \pi} e \bar{J} m a_{0} \int_{0}^{\infty} \frac{r d r}{\sqrt{d^{2}+r^{2}}} \int_{0}^{\pi} d \theta A^{\prime 2} \int_{0}^{2 \pi} d \varphi \frac{-\sin \varphi \hat{x}+\cos \varphi \hat{y}}{\sqrt{1+B \cos \varphi}}
$$

in the above:

$$
B(r, \theta, d) \equiv \frac{2 r d \sin \theta}{d^{2}+r^{2}}, \quad 0 \leq B \leq 1
$$

Obviously the cylindrical symmetry is restored when $B=0$. This happens for either small $d$ or large $d$, that is if the proton is too close or too far from the electron:

$$
\lim _{d->0} B=\lim _{d->\infty} B=0
$$

hence a relativistic motor effect may occur only in intermediate values of $d$. We notice that $\sin \theta \geq 0$ as $\theta \in[0, \pi]$, thus we are only interested in $B \geq 0$. We also notice that the maximal value of $B$ is for $\theta=\frac{\pi}{2}$ and for $r=d$ for which $B_{\max }=1$ as depicted in figure 2 .

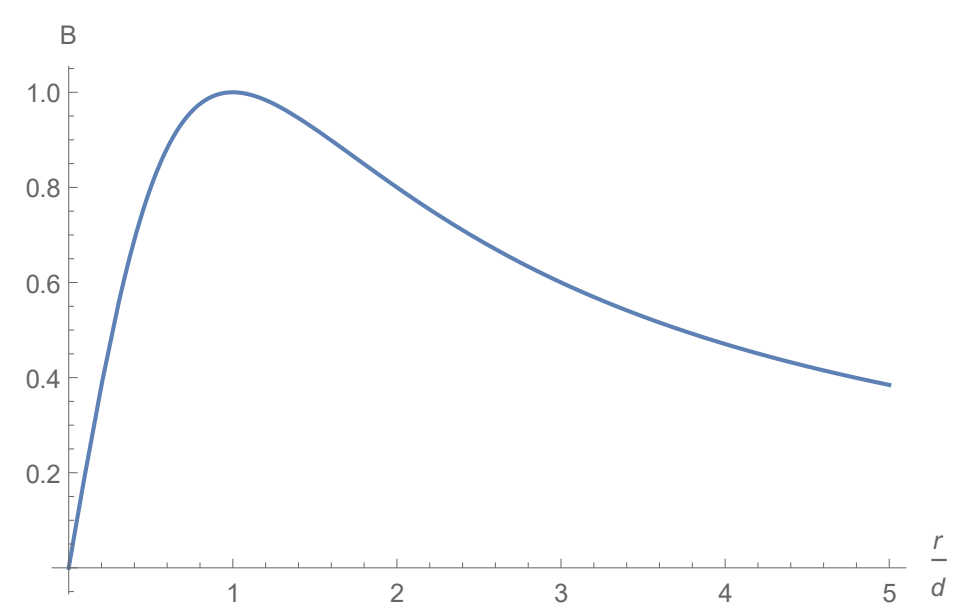

Figure 2. $B$ as function of $\frac{r}{d}$ for $\theta=\frac{\pi}{2}$. 
A triple integral needs to be evaluated in order to calculate the momentum, this cannot be done using only analytic techniques, however, at least part of the integration can be done analytically. Consider the integrals:

$$
I_{x}(B) \equiv \int_{0}^{2 \pi} d \varphi \frac{\sin \varphi}{\sqrt{1+B \cos \varphi}}, \quad I_{y}(B) \equiv \int_{0}^{2 \pi} d \varphi \frac{\cos \varphi}{\sqrt{1+B \cos \varphi}} .
$$

Integrating analytically we arrive at the following results:

$$
I_{x}(B)=0, \quad I_{y}(B)=\frac{4(1+B) \operatorname{Elliptic}_{E}\left(\frac{2 B}{1+B}\right)-4 \operatorname{Elliptic}_{K}\left(\frac{2 B}{1+B}\right)}{B \sqrt{1+B}} .
$$

in the above Elliptic $_{E}$ and Elliptic $_{K}$ are the elliptic $E$ and $K$ functions respectively. The function $I_{y}$ is an even function that is $I_{y}(B)=I_{y}(-B)$ and is depicted in figure 3 .

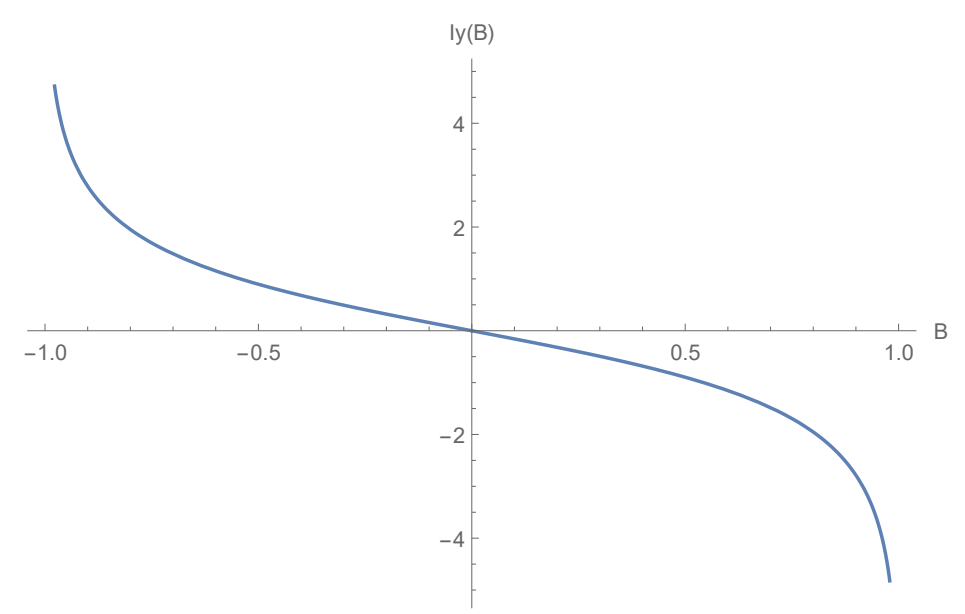

Figure 3. The even function $I_{y}(B)$.

We remark that since $B \geq 0$ it follows that $I_{y}(B) \leq 0$, thus only the right hand part of figure 3 is of interest. We also notice that $I_{y}(B)$ is monotonic decreasing function of $B$ hence the larger values of $B$ which are around $r=d, \theta=\frac{\pi}{2}$ will make the most significant contribution (in absolute terms) to $I_{y}(B)$. For $B=1$ this function is singular, however, the singularity will be integrated away when integrating over $\theta$.

We have obtained a counter intuitive result that the momentum is generated in the $y$ direction despite the fact that proton which breaks the symmetry is located in the $x$ direction, that is:

$$
\vec{P}=\frac{\mu_{0}}{4 \pi} e \bar{J} m a_{0} \int_{0}^{\infty} \frac{r d r}{\sqrt{d^{2}+r^{2}}} \int_{0}^{\pi} d \theta A^{\prime 2} I_{y}(B) \hat{y} .
$$

Next we define the function $F$ as follows:

$$
F \equiv \int_{0}^{\pi} d \theta A^{\prime 2} I_{y}(B)
$$

$F$ will become larger if both $A^{\prime 2}$ and $I_{y}$ are peaked at the same $r$ value, if the overlap is small so will be the relativistic momentum. We can thus write the momentum $\vec{P}$ in the form:

$$
\vec{P}=\frac{\mu_{0}}{4 \pi} e \bar{J} m a_{0} \int_{0}^{\infty} \frac{r d r}{\sqrt{d^{2}+r^{2}}} F \hat{y}=\frac{\mu_{0}}{4 \pi} e \bar{J} \frac{a_{0}^{3}}{d} m \int_{0}^{\infty} F \frac{\left(\frac{r}{a_{0}}\right) d\left(\frac{r}{a_{0}}\right)}{\sqrt{1+\left(\frac{r}{d}\right)^{2}}} \hat{y} .
$$

Thus $\vec{P}$ is given in the form:

$$
\vec{P}=\bar{P} \tilde{P} \hat{y}
$$


in which $\bar{P}$ is a dimensional constant independent of the quantum state of the electron:

$$
\bar{P}=\frac{\mu_{0}}{4 \pi} e \bar{J} \frac{a_{0}^{3}}{d}
$$

And $\tilde{P}$ is dimensionless, and depends on the quantum state.

$$
\tilde{P}_{n l m}=m \frac{n^{2}}{4} \int_{0}^{\infty} F_{n l m} \frac{r^{\prime} d r^{\prime}}{\sqrt{1+\left(\frac{r^{\prime}}{d^{\prime}}\right)^{2}}} .
$$

in the above we have made explicit the dependence on the quantum numbers and have made use of the normalized $r^{\prime}$ defined in equation (41) and also introduced a normalized $d^{\prime}$ using a similar definition:

$$
d^{\prime} \equiv \frac{2 d}{n a_{0}} .
$$

We also notice that since the hydrogen eigen state is given as a function of $r^{\prime}$ and also $B$ being dimensionless can be written in terms of dimensionless quantity:

$$
B=\frac{2 r^{\prime} d^{\prime} \sin \theta}{d^{\prime 2}+r^{\prime 2}} .
$$

it follows that $\tilde{P}_{n l m}$ only depends on the quantum state and $d^{\prime}$.

We shall now investigate the case $d=2 a_{0}$. In such a case the Hamiltonian of the electron is modified significantly and thus will have different eigenstates from the ones that are obtained for an isolated Hydrogen atom. Hence the eigen states described in equation (38) can only be considered as a superposition of the true eigen states. Nevertheless, it will suffice to study the relativistic momentum generated by the Hydrogen eigen states in this preliminary study. In this case:

$$
\bar{P}=\frac{\mu_{0}}{8 \pi} e \bar{J} a_{0}^{2} \simeq 5.310^{-29} \mathrm{Kg} \mathrm{m} / \mathrm{s}
$$

The velocity associate withe this momentum is:

$$
\bar{v}=\frac{\bar{P}}{2 m_{p}} \simeq 0.016 \mathrm{~m} / \mathrm{s},
$$

in we have taken into account both the mass of the proton and the mass of the Hydrogen atom. Hence for an unloaded relativistic motor we will obtain a velocity of:

$$
\vec{v}=\frac{\vec{P}}{2 m_{p}} \simeq 0.016 \tilde{P} \hat{y} \mathrm{~m} / \mathrm{s}
$$

We shall look at two cases of excited states.

\subsubsection{The case 211}

In the low excited state $n=2, l=1, m=1$ the square amplitude $A_{211}^{\prime 2}$ is depicted in figure 4 . 


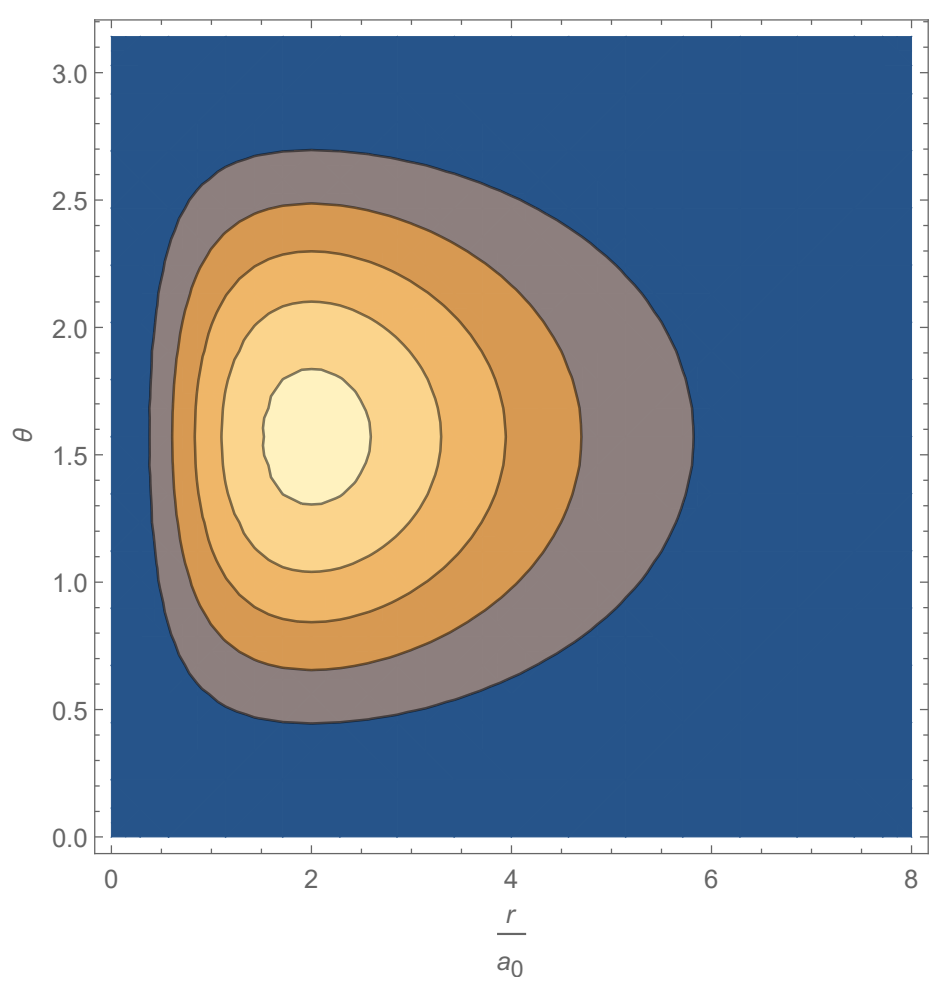

Figure 4. The square amplitude $A_{211}^{\prime 2}$.

It is seen that the function describes torii of equi-value. The function peaks at $\theta=\frac{\pi}{2}$ at $r=2 a_{0}$. A cross section of the same for $\theta=\frac{\pi}{2}$ is described in figure 5 :

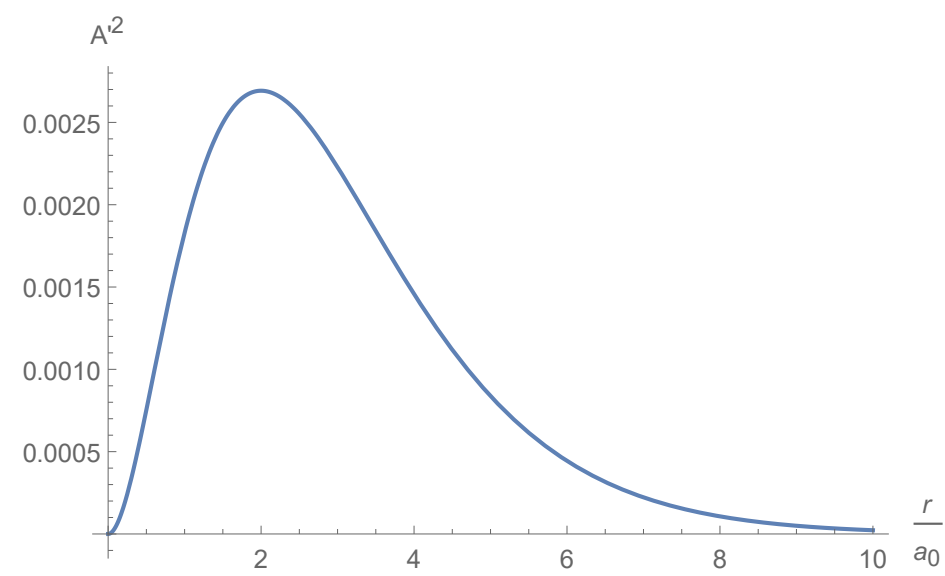

Figure 5. The square amplitude $A_{211}^{\prime 2}$ at $\theta=\frac{\pi}{2}$.

We are now able to evaluate numerically $F_{211}$ defined in equation (85) which is depicted in figure 6. 


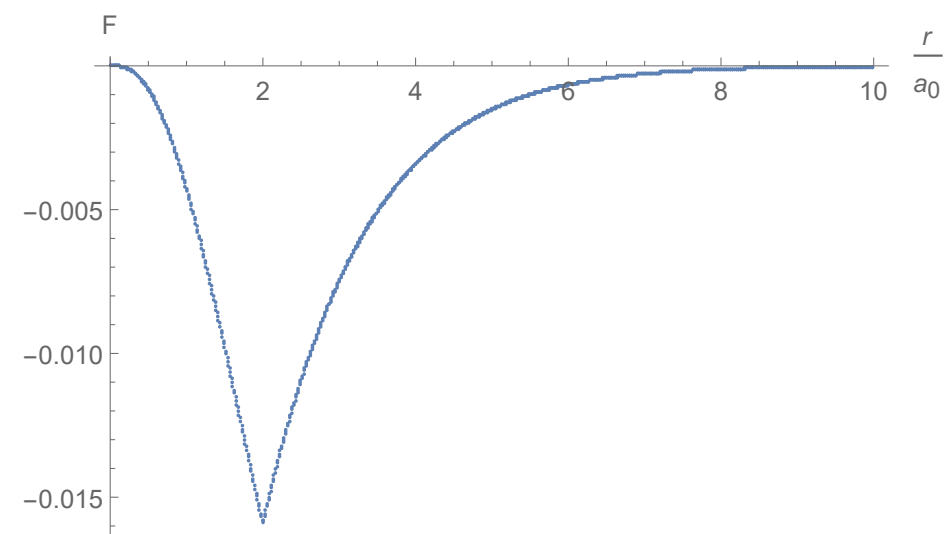

Figure 6. The function $F_{211}$.

Finally using $F_{211}$ we can calculate numerically $\tilde{P}_{211}$ using equation (89) and obtain the value:

$$
\tilde{P}_{211} \simeq-0.047
$$

Hence according to equation (87) and equation (94) we obtain:

$$
\vec{P}_{211} \simeq-2.4710^{-30} \hat{y} \mathrm{~kg} \mathrm{~m} / \mathrm{s} \quad \vec{v}_{211}=\simeq-7.410^{-4} \hat{y} \mathrm{~m} / \mathrm{s}
$$

Obviously even if many such systems are accumulated the total mass will grow accordingly and the final achievable velocity will be rather slow.

\subsubsection{The case 433}

In the high excited state $n=4, l=3, m=3$ the square amplitude $A_{433}^{\prime 2}$ is depicted in figure 7.

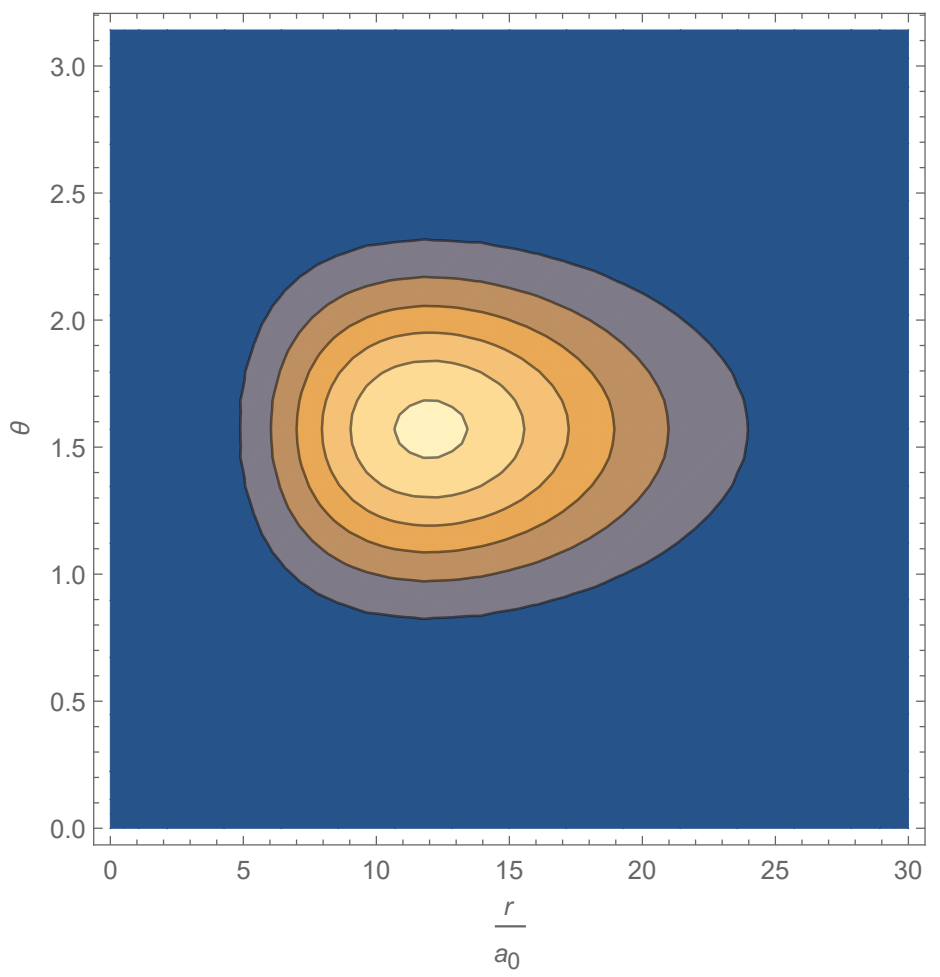

Figure 7. The square amplitude $A_{433}^{\prime 2}$. 
It is seen that the function describes torii of equi-value. The function peaks at $\theta=\frac{\pi}{2}$ at $r \simeq 12 a_{0}$, hence the Hydrogen atom becomes much larger. A cross section of the same for $\theta=\frac{\pi}{2}$ is described in figure 8 :

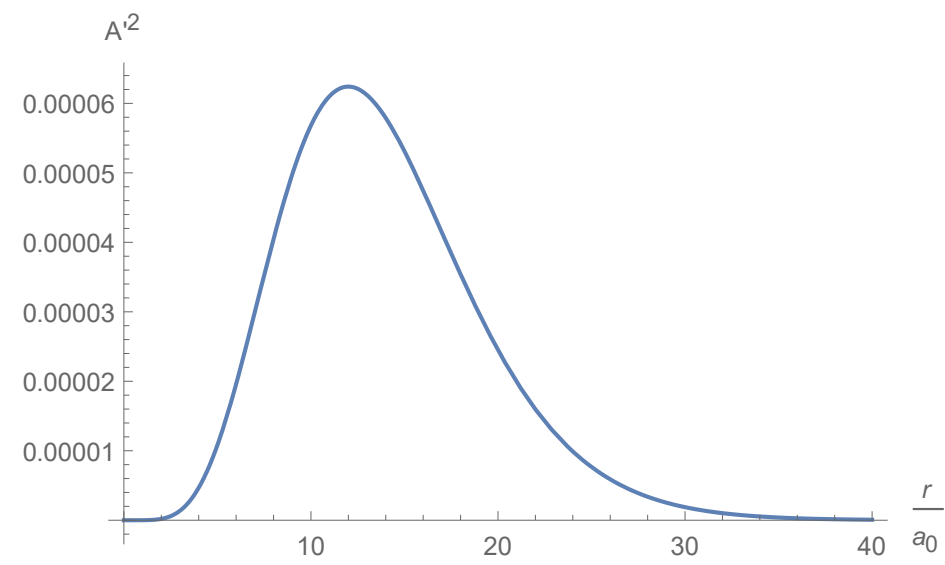

Figure 8. The square amplitude $A_{433}^{\prime 2}$ at $\theta=\frac{\pi}{2}$.

We are now able to evaluate numerically $F_{433}$ defined in equation (85) which is depicted in figure 9.

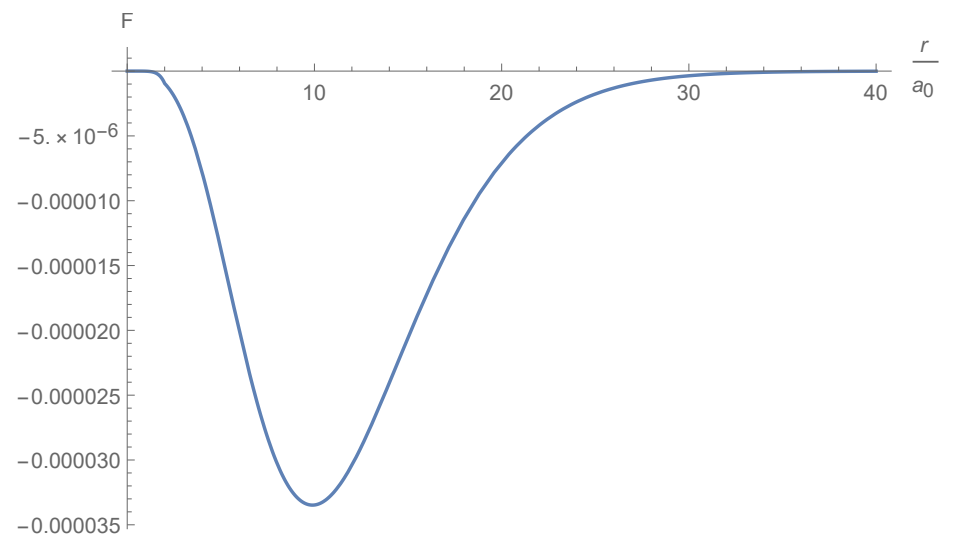

Figure 9. The function $F_{433}$.

Finally using $F_{433}$ we can calculate numerically $\tilde{P}_{433}$ using equation (89) and obtain the value:

$$
\tilde{P}_{433} \simeq-0.002 .
$$

this is surprisingly smaller than $P_{211}$, but can be understood due to the small overlap between $I_{y}(B)$ and $A_{433}^{\prime 2}$. Now according to equation (87) and equation (94) we obtain:

$$
\vec{P}_{433} \simeq-1.0510^{-31} \hat{y} \mathrm{~kg} \mathrm{~m} / \mathrm{s} \quad \vec{v}_{433}=\simeq-3.110^{-5} \hat{y} \mathrm{~m} / \mathrm{s} .
$$

Obviously even if many such systems are accumulated the total mass will grow accordingly and the final achievable velocity will be rather slow. The situation can be some what improved if the overlap between $I_{y}(B)$ and $A_{433}^{\prime 2}$ is better. This will happen if we choose $d \simeq 12 a_{0}$, however, a detailed calculation gives in this case:

$$
\vec{P}_{433} \simeq-2.710^{-31} \hat{y} \mathrm{~kg} \mathrm{~m} / \mathrm{s} \quad \vec{v}_{433}=\simeq-8.010^{-5} \hat{y} \mathrm{~m} / \mathrm{s} .
$$

this is better than before but worse than what is obtained in a low excited state 211 . 


\subsubsection{A nano relativistic motor}

A similar analysis was attempted for a very high excited state $n=11, l=10, m=$ 10, in this case which appears in Humphrey's series [29] $A_{11}^{\prime 2}{ }_{10}{ }_{10}$ peaks at $110 a_{0} \simeq$ 5.8 nano $\mathrm{m}$, which thus justly deserves the name a nano relativistic motor. Choosing $d$ to coincide with the above value we obtain, however, after a detailed calculation the values:

$$
\vec{P}_{111010} \simeq-2.010^{-32} \hat{y} \mathrm{~kg} \mathrm{~m} / \mathrm{s} \quad \vec{v}_{111010}=\simeq-5.910^{-6} \hat{y} \mathrm{~m} / \mathrm{s} .
$$

this does not qualify as an improvement.

\section{Discussion}

In a previous paper, we have shown that, in general, Newton's third law is not compatible with the principles of special relativity and the total force on a two charged body system is not zero. Still, momentum is conserved if one takes the field momentum into account, and the same is true for energy.

The dielectric strength's and current density limitations of macroscopic bodies led us to consider the microscopic realm in which charge densities and current densities are considerably higher.

The main results of this paper are the possibility of implementing a relativistic motor in the atomic and nano scales. It is shown that a Hydrogen atom whether in a ground or excited state does not produce any momentum according to the relativistic motor equation.

Thus two configurations are studied, in one the Hydrogen electron is put in a wave packet state rather than in an eigen state. In this approach one can reach high momentum which means high velocity for both a loaded and an unloaded engine. However, for a considerable momentum to be realized the wave packet must be highly localized to a subatomic scale. To achieve even higher momentum, sub nuclear scales are required. In order to change the state of Hydrogen atom from the ground state to any desired state a suitable Hamiltonian is to be constructed. Thus the desired wave packet can only be achieve by an appropriate electromagnetic field which must be non trivial.

A second configuration involves symmetry breaking by adding an additional proton to the hydrogen atom. For simplicity we assume that despite this addition the eigenstates of the Hydrogen atom are still valid. This approach yields low momenta and low velocities for high and low excited states.

\section{Conclusion}

To conclude we remark that despite the possibility in principle to construct a working relativistic motor, this is not a trivial task and involves the creation of a highly localized wave packet. Thus in a study which is not a merely preliminary as this one, the electromagnetic field needed to achieve this goal must be specified. And we notice that the wave packet should not only be created but also maintained for an engine that may become useful.

Additional directions for future studies which are arise from this paper include:

1. The analysis of a relativistic motor of which its components move also at relativistic speeds and not just the electromagnetic signals transmitted between the components. The need for this arises as the electron studied in the current paper moves at relativistic speeds.

2. For the same reason an analysis of the relativistic motor in the frame work of a Dirac theory is required. The Schrödinger equation and even the Pauli equation are not appropriate for the study of an electron at relativistic speeds.

3. For the symmetry breaking approach, despite the fact that it shows little promise for a useful relativistic motor, one should use true eigenstates (and not eigen- 
states of the Hydrogen atom which are clearly not appropriate). Perhaps for those eigenstates the results will be more encouraging.

\section{References}

1. Miron Tuval \& Asher Yahalom "Newton's Third Law in the Framework of Special Relativity" Eur. Phys. J. Plus (11 Nov 2014) 129: 240 DOI: 10.1140/epjp/i2014-14240-x. (arXiv:1302.2537 [physics.gen-ph]).

2. Miron Tuval and Asher Yahalom "A Permanent Magnet Relativistic Engine" Proceedings of the Ninth International Conference on Materials Technologies and Modeling (MMT-2016) Ariel University, Ariel, Israel, July 25-29, 2016.

3. Asher Yahalom "Retardation in Special Relativity and the Design of a Relativistic Motor". Acta Physica Polonica A, Vol. 131 (2017) No. 5, 1285-1288. DOI: 10.12693/APhysPolA.131.1285

4. Miron Tuval and Asher Yahalom "Momentum Conservation in a Relativistic Engine" Eur. Phys. J. Plus (2016) 131: 374. DOI: 10.1140/epjp/i2016-16374-1

5. Asher Yahalom "Preliminary Energy Considerations in a Relativistic Engine" Proceedings of the Israeli-Russian Bi-National Workshop "The optimization of composition, structure and properties of metals, oxides, composites, nano - and amorphous materials", page 203-213, 28 - 31 August 2017, Ariel, Israel.

6. S. Rajput and A. Yahalom, "Preliminary Magnetic Energy Considerations in a Relativistic Engine: Mutual Inductance vs. Kinetic Terms" 2018 IEEE International Conference on the Science of Electrical Engineering in Israel (ICSEE), Eilat, Israel, 2018, pp. 1-5. doi: 10.1109/ICSEE.2018.8646265

7. S. Rajput and A. Yahalom, "Material Engineering and Design of a Relativistic Engine: How to Avoid Radiation Losses". Advanced Engineering Forum ISSN: 2234-991X, Vol. 36, pp 126-131. Submitted: 2019-06-16, Accepted: 2020-05-18, Online: 2020-06-17. (02020 Trans Tech Publications Ltd, Switzerland.

8. Shailendra Rajput, Asher Yahalom \& Hong Qin "Lorentz Symmetry Group, Retardation and Energy Transformations in a Relativistic Engine" Symmetry 2021, 13, 420. https:/ / doi.org/10.3390/sym13030420.

9. A. Einstein, "On the Electrodynamics of Moving Bodies", Annalen der Physik 17 (10): 891-921, (1905).

10. J.C. Maxwell, "A dynamical theory of the electromagnetic field" Philosophical Transactions of the Royal Society of London 155: 459-512 (1865).

11. J. D. Jackson, Classical Electrodynamics, Third Edition. Wiley: New York, (1999).

12. R. P. Feynman, R. B. Leighton \& M. L. Sands, Feynman Lectures on Physics, Basic Books; revised 50th anniversary edition (2011).

13. O. Heaviside, "On the Electromagnetic Effects due to the Motion of Electrification through a Dielectric" Philosophical Magazine, (1889).

14. I. Newton, Philosophiae Naturalis Principia Mathematica (1687).

15. H. Goldstein, C. P. Poole Jr. \& J. L. Safko, Classical Mechanics, Pearson; 3 edition (2001).

16. G. D'Abramo, "On apparent faster-than-light behavior of moving electric fields" Eur. Phys. J. Plus, 1363 (2021) 301. DOI: https://doi.org/10.1140/epjp/s13360-021-01283-5

17. M. Mansuripur, "Trouble with the Lorentz Law of Force: Incompatibility with Special Relativity and Momentum Conservation" PRL 108, 193901 (2012).

18. D. J. Griffiths \& M. A. Heald, "Time dependent generalizations of the Biot-Savart and Coulomb laws" American Journal of Physics, 59, 111-117 (1991), DOI:http:/ /dx.doi.org/10.1119/1.16589

19. Jefimenko, O. D., Electricity and Magnetism, Appleton-Century Crofts, New York (1966); 2nd edition, Electret Scientific, Star City, WV (1989).

20. Rajput, Shailendra, and Asher Yahalom. 2021. "Newton's Third Law in the Framework of Special Relativity for Charged Bodies" Symmetry 13, no. 7: 1250. https://doi.org/10.3390/sym13071250

21. Dielectric Strength of Air - the Physics Fact book https:/ /hypertextbook.com/facts/2000/AliceHong.shtml

22. Stefan Giere, Michael Kurrat \& Ulf Schümann, HV Dielectric Strength of Shielding Electrodes in Vacuum Circuit-Breakers, $20^{\text {th }}$ International Symposium on Discharges and Electrical Insulation in Vacuum - Tours, France - June 30 - July 5, 2002

23. Markus Gabrysch "Electronic properties of diamond". el.angstrom.uu.se. Retrieved 2013-08-10.

24. L. Hsi-wen, T. Yu-Chong, Parylene-based electret power generators, J. Micromech. Microeng. 18 (10) (2008) 104006.

25. Jung, S.-G. et al. Enhanced critical current density in the pressure-induced magnetic state of the high-temperature superconductor FeSe. Sci. Rep. 5, 16385; doi: 10.1038/srep16385 (2015).

26. Peebles, P. Z. Probability, Random Variables and Random Signal Principles, McGraw Hill, New York, NY, USA (2001).

27. E. Schrödinger, Ann. d. Phys. 81109 (1926). English translation appears in E. Schrödinger, Collected Papers in Wave Mechanics (Blackie and Sons, London, 1928) p. 102

28. P.R. Holland The Quantum Theory of Motion (Cambridge University Press, Cambridge, 1993)

29. Humphreys, C.J. (1953), "The Sixth Series in the Spectrum of Atomic Hydrogen", Journal of Research of the National Bureau of Standards, 50: 1, doi:10.6028/jres.050.001. 